\title{
Discalculia do Desenvolvimento: Avaliação da Representação Numérica pela ZAREKI-R ${ }^{1}$
}

\author{
Paulo Adilson da Silva ${ }^{2}$ \\ Flávia Heloísa dos Santos ${ }^{3}$ \\ Universidade Estadual Paulista “Júlio de Mesquita Filho”, UNESP, Campus Assis
}

\begin{abstract}
RESUMO - O presente estudo investigou aspectos da representação numérica (processamento numérico e cálculo) e memória operacional de crianças com transtornos de aprendizagem. Participaram 30 crianças de idade entre 9 e 10 anos, ambos os gêneros, divididas em dois grupos: sem dificuldade em aritmética (SDA; N=11) e com dificuldade em aritmética (CDA; $\mathrm{N}=19$ ), avaliadas pela ZAREKI-R, Matrizes Coloridas de Raven, o Blocos de Corsi e o BCPR. Crianças CDA exibiram escores levemente mais baixos que as SDA quanto ao nível intelectual e nos Blocos de Corsi. Na ZAREKI-R apresentaram prejuízo nos subtestes ditado de números, cálculo mental, problemas aritméticos e total. Crianças CDA apresentaram déficits específicos em memória operacional visuoespacial e comprometimento em processamento numérico e cálculo, compatível com discalculia do desenvolvimento.
\end{abstract}

Palavras-chave: Discalculia do Desenvolvimento; Matemática; Memória Operacional; Neuropsicologia; Transtornos de Aprendizagem.

\section{Developmental Dyscalculia: Assessment of Number Representation by the ZAREKI-R}

\begin{abstract}
This study investigated which aspects of number processing and calculation and working memory are related to arithmetic deficits. The participants were children aged from 9 to 10 years, both gender, divided in two groups based on their calculation score: a group without arithmetic disability (SDA; N=11) and with arithmetic disability (CDA; N=19); assessed by the instruments: ZAREKI-R, Raven's Coloured Progressive Matrices, Block Span, Digit Span, and BCPR (pseudowords repetition). CDA children exhibited slightly lower scores than SDA on intellectual level and Block Span, and deficits in dictation of numbers, mental calculation, and problem solving tasks of the ZAREKI-R. The results indicate that CDA children have specific deficits in visuospatial working memory and performed poorly in number representation tasks, which fulfill criteria for Developmental Dyscalculia.
\end{abstract}

Keywords: Developmental Dyscalculia; Mathematic; Working memory; Neuropsychology; learning disabilities.

Há uma capacidade inata para habilidades quantitativas que inclui uma compreensão implícita de numerosidade, ordinalidade, contagem e aritmética simples (Geary, 1995), a qual constitui um modo de processamento não simbólico de quantidades, baseado em subtização e aproximação (Feigenson, Dehaene \& Spelke, 2004). Essa capacidade se desenvolve

1 Agradecimentos: À FAPESP que financiou este estudo, processo 05/60375-1. À Secretaria Municipal de Educação do Município de Assis-SP; às diretoras e coordenadoras pedagógicas das escolas municipais. Às crianças e aos seus pais que participaram do estudo. À Ana Luiza Ribeiro Pereira Dias, Bruna Pachoalini, Juliana Molina e Michele Cândida Frigério que auxiliaram na coleta de dados.

2 Observações: Este estudo é o resultado da Iniciação Científica de P.A. Silva e foi conduzido junto ao Laboratório de Neuropsicologia do Departamento de Psicologia Experimental e do Trabalho, Universidade Estadual Paulista - Faculdade de Ciências e Letras - Campus de Assis. Resultados apresentados no I Congresso Multidisciplinar de Transtornos de Aprendizagem (São Paulo, 2008). I Congresso IBRO/ LARC de Neurociências da América Latina, Caribe e Península Ibérica (Armação de Búzios, 2008)

3 Endereço para correspondência: Universidade Estadual Paulista Faculdade de Ciências e Letras - Campus de Assis. Departamento de Psicologia Experimental e do Trabalho, Laboratório de Neuropsicologia. Avenida Dom Antônio, 2100. Assis, SP. CEP 19806-900. Fone: (18) 3302-5902 Fax: (18) 3302-5804.E-mail: flaviahs@assis.unesp.br. gradualmente no decorrer dos anos pré-escolares, juntamente com a linguagem e sua estruturação (Geary, 2000). Com a inserção no Ensino Fundamental, as crianças aprendem a representar os números por meio do processamento simbólico (p.ex., palavras, numerais, ideogramas), o que permite a manipulação e comparação de quantidades precisas (Mundy \& Gilmore, 2009). Uma vez que as habilidades quantitativas adquirem características culturais (Geary, 2000; von Aster \& Shalev, 2007), fatores linguísticos, culturais e pedagógicos têm diferentes efeitos nos diversos componentes das habilidades matemáticas e podem, em alguns casos, afetar a aprendizagem escolar (Dellatolas, von Aster, Braga, Meier, \& Deloche, 2000).

Segundo McCloskey, Caramazza e Basili (1985) a representação numérica baseia-se em dois componentes: o processamento numérico e o cálculo. O processamento numérico englobaria tanto compreensão numérica, ou seja, o entendimento da natureza dos símbolos numéricos e de suas quantidades quanto a produção numérica, isto é, a escrita, leitura e contagem de números ou objetos. Em outras palavras, o processamento numérico propicia a aquisição de uma série de propriedades numéricas, as quais subsidiam o cálculo. Por outro lado, o cálculo se refere ao processamento dos símbolos (p. ex.,,$+- \times$ ou $\div$ ) ou palavras (p. ex. mais, menos, vezes, dividir) operacionais, à recuperação junto à memória de 
longo prazo de fatos aritméticos básicos (p. ex. tabuada) e à execução de procedimentos de cálculos aritméticos.

Dehaene (2001) difundiu o conceito de 'senso numérico', uma habilidade universal para representar e manipular magnitudes numéricas não-verbais em uma 'linha numérica mental' orientada espacialmente. À medida que a criança tem experiências aritméticas, o desenvolvimento da 'linha numérica mental' se automatiza, aumentando espacialmente a imagem mental de números ordinais, processo que depende da interação entre o entendimento da magnitude e das propriedades simbólicas e espaciais dos números. A aquisição do senso numérico culminaria no pensamento aritmético propriamente dito e dependeria também das funções cognitivas que se desenvolvem durante a pré-escola e os primeiros anos de escolarização, de aspectos genéticos, bem como de habilidade de memória operacional e simbolização numérica (von Aster \& Shalev, 2007).

De acordo com Baddeley (1986) a memória operacional é a capacidade de manipular e reter informações por curtos períodos de tempo e está associada à resolução de problemas, entre outros. Essa habilidade também é responsável pelo resgate das informações em conjunto com a memória de longo prazo. Conforme o modelo proposto por Baddeley e Hitch (1974) a memória operacional é formada pelo menos por três componentes: o esboço visuoespacial, para armazenamento de informações visuoespaciais; a alça fonológica, para retenção de informações verbais; e o executivo central, um controlador atencional responsável pela manipulação da informação. Em recente revisão (Baddeley, 2000) incluiu o componente retentor episódico que integraria as informações em um episódio único e consciente.

Há evidências de associação entre memória operacional e habilidades aritméticas durante o desenvolvimento (Adams \& Hitch, 1997; Nöel, 2005; Rotzer \& cols., 2009; Schuchardt, Maehler, \& Hasselhorn, 2008, Swanson, 1993). Conforme Swanson (1993) crianças com Transtornos de Aprendizagem (TA) apresentam prejuízos generalizados em memória operacional. Para McLean e Hitch (1999) crianças com dificuldades de aprendizagem em aritmética, especificamente, apresentam déficits em esboço visuoespacial e aspectos do executivo central responsáveis pela mediação com a memória de longo prazo. Schuchardt e cols. (2008) observaram que crianças com diferentes transtornos de aprendizagem apresentam distintos perfis de comprometimento em memória operacional, de forma que crianças com déficit em aritmética apresentam prejuízo seletivo para o esboço visuoespacial.

Segundo Dias, Enumo, Turini e Ferrão (2003) problemas relacionados à aquisição das habilidades de aritmética, escrita e leitura podem ser observados no Ensino Fundamental brasileiro. Ciasca (1994) aponta que $30 \%$ a $40 \%$ das crianças podem apresentar alguma dificuldade de aprendizagem nos primeiros anos do Ensino Fundamental. Contudo, a prevalência de TA específicos corresponde em média de 2 a $10 \%$ da população (APA, 2002). Diferentemente das dificuldades de aprendizagem, os TA têm como característica um baixo desempenho em testes padronizados para escrita, leitura e aritmética em comparação a crianças de mesma idade, nível de inteligência e escolarização (APA, 2002) que não pode ser justificado por falta de oportunidade de aprendizagem, falhas pedagógicas, déficits sensoriais ou lesões adquiridas (OMS, 1993). Embora estudos de diferentes países revelem uma prevalência similar entre os tipos de TA, nota-se que o número de pesquisas relativas ao prejuízo em aritmética é muito menor do que o de pesquisas sobre déficits em leitura, por exemplo (Haskell, 2000, Rubinsten \& Henik, 2009).

Dentre os TA, o 'Transtorno de Matemática' descrito pelo DSM-IV-TR (APA, 2002), referido no CID-10 (OMS, 1993) como 'Transtorno específico de habilidades aritméticas' $\mathrm{e}$ internacionalmente conhecido como Discalculia do Desenvolvimento (DD) caracteriza-se como um transtorno específico que afeta a aquisição normal das habilidades aritméticas em crianças com inteligência normal e adequadas oportunidades de escolarização (Gross-Tsur, Manor, \& Shalev, 1996; Shalev, 2004; Shalev \& von Aster, 2008; von Aster, Schweiter, \& Weinhold Zulauf, 2007). Evidências genéticas, neuroanatômicas e epidemiológicas indicam que a DD é uma desordem de etiologia neurobiológica, embora fatores ambientais possam potencializar sua manifestação (Dellatolas \& cols., 2000; Haskell, 2000; Rotzer \& cols., 2009; Shalev, 2004; von Aster \& Shalev, 2007). Sua prevalência varia de $3-6,5 \%$, afetando meninos e meninas nas mesmas proporções, contudo, a maioria dos casos de DD apresenta comorbidades, como a dislexia e o TDAH (Gross-Tsur \& cols., 1996; Koumoula \& cols., 2004; Lewis, Hitch, \& Walker, 1994; Shalev, Auerbach, Manor, \& Gross-Tsur, 2000; von Aster \& cols., 2007). Além disso, estudos longitudinais mostraram que a DD é uma desordem persistente, se estendendo até além da adolescência (Shalev, Manor, Auerbach, \& Gross-Tsur, 1998; Shalev, Manor, \& Gross-Tsur, 2005).

O status socioeconômico das famílias mostrou ter influência sobre o desempenho aritmético (Dellatolas \& cols., 2000, Koumoula \& cols., 2004). Em um estudo transcultural realizado no Brasil, Suíça e França, crianças brasileiras de menor nível socioeconômico apresentaram baixo desempenho em cálculo mental e em tarefas que envolviam o processamento de números na forma escrita: produção (ditado de números) e compreensão numérica (leitura e comparação de números) (Dellatolas \& cols. 2000)

A avaliação do cálculo, por tarefas como cálculo mental e problemas aritméticos, apresenta peculiaridades. No primeiro, as operações aritméticas são estruturadas enquanto no segundo a criança deve ouvir e interpretar os aspectos sintáticos e simbólicos do enunciado, discriminar as informações relevantes, identificar a incógnita e a sua posição e, então, realizar as operações matemáticas apropriadas à resolução do problema (Haydu, Costa, \& Pullin, 2006). Prejuízos na resolução de problemas aritméticos apresentados na forma de sentença (word problems) podem decorrer de falhas: 1) na produção numérica a partir do enunciado (McCloskey \& cols., 1985); 2) no processamento dos símbolos ou palavras operacionais e na execução de procedimentos de cálculos aritméticos (McCloskey \& cols., 1985); 3) na identificação da posição da incógnita na sentença linguística (Haydu \& cols., 2006; Fayol, 1996; Chahon, 2006); e 4) na memória operacional (Baddeley, 1986; Schuchardt \& cols, 2008).

A Bateria de Testes Neuropsicológicos para Processamento Numérico e Cálculo em Crianças, versão revisada, conhecida como ZAREKI-R (von Aster \& Dellatolas, 2006), é um instrumento reconhecido internacionalmente que visa a identificar e especificar o perfil das habilidades matemáticas em crianças no domínio do cálculo e do processamento de números (Koumoula \& cols., 2004). 
O presente estudo tem como objetivo identificar quais aspectos da representação numérica e memória operacional explicariam prejuízos em aritmética de crianças com Transtornos de Aprendizagem (TA) utilizando a ZAREKI-R (von Aster \& Dellatolas, 2006), instrumento recomendado para a avaliação da DD (Shalev \& von Aster, 2008), e medidas de memória operacional visuoespaciais e fonológica. Os autores têm como hipótese que crianças do grupo CDA (com dificuldade em aritmética), além de prejuízos em cálculos, apresentarão um comprometimento em tarefas que avaliam o processamento numérico, pois este constitui a base para o cálculo. Além disso, espera-se que estas mesmas crianças apresentem prejuízos em relação aos componentes esboço visuoespacial e executivo central da memória operacional. Portanto, supõe-se que as crianças CDA apresentarão prejuízos globais em representação numérica, mas prejuízos específicos em memória operacional.

\section{Método}

Este estudo foi aprovado pelo Comitê de Ética da Universidade Estadual Paulista "Júlio de Mesquita Filho", da FCL - Campus de Assis. O Termo Livre e Esclarecido foi assinado por todos os pais ou responsáveis das crianças que participaram do estudo.

\section{Participantes}

Foram selecionadas 30 crianças, 14 meninas, de idade entre 9 e 10 anos, com nível intelectual normal - percen- til $=51,3 \pm 16,7$ (Raven, 1992), balanceadas entre as $3^{\mathrm{a}}$ e $4^{\mathrm{a}}$ séries de duas escolas municipais da cidade de Assis-SP. Os critérios para inclusão foram (a) a participação em salas de reforço devido às dificuldades de aprendizagem detectadas pelos professores por meio do desempenho escolar, (b) mensuração objetiva das dificuldades de aprendizagem utilizando o escore bruto total do Teste de Desempenho Escolar - TDE ( $3^{\mathrm{a}}$ Série, $\mathrm{M}=80,5 \pm 24,5 ; 4^{\mathrm{a}}$ Série, $\mathrm{M}=88,7$ $\pm 17,5$; Stein, 1994), que avalia a aprendizagem escolar associada à leitura, escrita ou aritmética. As médias obtidas para $3^{\mathrm{a}}$ e $4^{\mathrm{a}}$ Séries se encontravam respectivamente a dois e três desvios-padrão abaixo do esperado para estas séries e, além disso, seus escores correspondiam aos de crianças de $1^{\mathrm{a}}$ e $2^{\mathrm{a}}$ Séries, respectivamente, denotando um prejuízo consistente com TA (APA, 2002). Os critérios de exclusão foram evidências de patologias neurológicas, psiquiátricas ou de outra ordem, subnutrição, reprovação escolar, distúrbios de comportamento e uso de substâncias psicoativas, aferidos na ocasião da anamnese. Posteriormente, os participantes foram divididos em dois grupos, a partir do desempenho no Teste de Aritmética do TDE: CDA (com dificuldade em aritmética) e SDA (sem dificuldade em aritmética). Todas as crianças CDA apresentavam classificação 'inferior' para o Teste de Aritmética, o que constituiu o critério (c).

A Escala para Avaliação do Status Sócio-Econômico da Associação Brasileira dos Institutos de Pesquisa de Mercado - ABIPEME (Almeida \& Wickerhauser, 1991) foi utilizada para avaliar o status socioeconômico. Os dois grupos foram classificados como "C", que é equivalente à classe média. A Tabela 1 mostra os resultados demográficos e neuropsicológicos dos dois grupos.

Tabela 1. Dados demográficos e desempenho neuropsicológico [Média (DP)] por grupos.

\begin{tabular}{|c|c|c|c|c|}
\hline & $\operatorname{SDA}(\mathrm{N}=11)$ & CDA $(\mathrm{N}=19)$ & Teste Estatístico & $\mathrm{p}$ \\
\hline Idade $[\mathrm{N}](9 / 10)$ & $7 / 4$ & $9 / 10$ & & \\
\hline ABIPEME & $35,27(11,78)$ & $42,38(14,67)$ & $\mathrm{t}=-1,35$ & 0,18 \\
\hline Sexo $[\mathrm{N}]$ (meninos/meninas) & $5 / 6$ & $11 / 8$ & & \\
\hline MPC & $61,72(19,20)$ & $45,26(11,72)$ & $\mathrm{t}=2,92$ & $0,01 *$ \\
\hline Teste de Aritmética do TDE & $18,72(3,34)$ & $12,15(4,05)$ & $t=4,53$ & $<0,0001$ \\
\hline Teste de Leitura do TDE & $57,27(8,56)$ & $49,94(12,60)$ & $t=1,70$ & 0,09 \\
\hline Teste de Escrita do TDE & $21,36(7,31)$ & $14,73(6,74)$ & $\mathrm{t}=2,51$ & 0,01 \\
\hline Escore Total do TDE & $97,36(16,18)$ & $76,84(20,95)$ & $\mathrm{t}=2,79$ & 0,01 \\
\hline BCPR & $36,18(4,09)$ & $35,26(1,96)$ & $\mathrm{t}=0,83$ & 0,41 \\
\hline Blocos de Corsi & & & $F(1,28)=6,11$ & 0,02 \\
\hline Ordem Direta & $5,54(0,93)$ & $4,68(0,88)$ & & \\
\hline Ordem Inversa & $5,00(1,34)$ & $4,05(1,39)$ & & \\
\hline Span de Dígitos & & & $F(1,28)=1,697$ & 0,20 \\
\hline Ordem Direta & $4,72(0,90)$ & $3,94(0,91)$ & & \\
\hline Ordem Inversa & $2,90(0,54)$ & $3,05(0,84)$ & & \\
\hline
\end{tabular}

Legenda: SDA - sem dificuldade em aritmética; CDA - com dificuldade em aritmética; ABIPEME - Avaliação do Status Sócio-Econômico da Associação Brasileira dos Institutos de Pesquisa de Mercado; MPC - Matrizes Progressivas Coloridas de Raven, Escala Especial; BCPR - Teste de Repetição de Pseudopalavras para crianças brasileiras; TDE - Teste de Desempenho Escolar. 


\section{Procedimentos}

Após a autorização da Secretaria Municipal de Educação de Assis-SP, foi estabelecido contato com duas escolas municipais que ofereciam proposta similar de reforço escolar às crianças com dificuldades de aprendizagem e que aceitaram colaborar com o estudo. Nestas escolas as crianças foram identificadas pelos professores, contudo, não apresentavam diagnósticos de transtornos do desenvolvimento psicológico (OMS, 1993) ou de outra natureza. Foram marcadas reuniões com os pais ou responsáveis nas respectivas escolas para as devidas explicações sobre os procedimentos e objetivos da pesquisa. Em seguida os que demonstraram interesse e consentiram por escrito com a avaliação foram convocados para uma entrevista de anamnese, na qual forneceram dados relevantes sobre nascimento e desenvolvimento de seus filhos.

Os dados obtidos nesta entrevista de anamnese foram contrastados com os critérios de inclusão e exclusão pré-estabelecidos para que a criança pudesse participar das etapas seguintes da pesquisa. Em seguida as avaliações neuropsicológicas foram iniciadas. Cada avaliação foi realizada individualmente em sala apropriada, livre de ruídos ou estímulos que pudessem distrair a criança. A aplicação dos testes foi feita em ordem semirrandômica (intercalando tarefas verbais e não-verbais) e foi realizada em uma sessão única de aproximadamente 120 minutos, com intervalo de 20 minutos para evitar o efeito de fadiga. As avaliações foram realizadas no período de outubro de 2006 a abril de 2007.

\section{Materiais}

Matrizes progressivas coloridas de Raven: Escala especial (Raven, 1992): este teste de inteligência é constituído por três séries de doze itens (A, AB e B), que aumentam progressivamente em dificuldade, com duração aproximada de 15 minutos.

Teste de Desempenho Escolar - TDE (Stein, 1994): este teste tem como objetivo avaliar o desempenho acadêmico entre crianças de $1^{\mathrm{a}}$ a $6^{\mathrm{a}}$ séries. Ele avalia habilidades de escrita, leitura e aritmética. Cada um dos subtestes apresenta uma escala de itens que aumenta gradativamente em complexidade. Tem duração de aproximadamente 40 minutos e apresenta três testes. Teste de Escrita: a criança deve escrever o seu próprio nome e mais 34 palavras isoladas apresentadas sob forma de ditado. Teste de Aritmética: a criança deve dar uma solução oral para três problemas e calcular mais 35 operações aritméticas dadas por escrito. Teste de Leitura: a criança deve ler 70 palavras isoladas dos seus contextos.

BCPR - Teste de Repetição de Pseudopalavras para crianças brasileiras (Santos \& Bueno, 2003): É dito para cada criança no começo do teste que ele/ela irá ouvir algumas "palavras inventadas engraçadas" que ela vai ter que tentar repetir em voz alta. Os itens são apresentados numa sequência constante para todos os sujeitos, ditas pelo experimentador com a boca escondida por uma folha de papel para prevenir a leitura labial. São dados três segundos para a criança tentar repetir a palavra. $\mathrm{O}$ experimentador fala as próximas pseudopalavras na sequência depois de permitir uma tentativa de repetição do item anterior. Este teste avalia o componente alça fonológica da memória operacional, relacionado a informações verbais. Tem duração máxima de cinco minutos.

Blocos de Corsi: Um tabuleiro de 26x32 centímetros contendo 10 blocos de $4 \times 4 \times 4$ centímetros cada, fixos, arranjados em uma disposição específica é apresentado à criança. $\mathrm{O}$ examinador toca certo número de blocos (mínimo de 2 , máximo de 8 unidades) numa sequência pré-estabelecida. $\mathrm{Na}$ ordem direta pede-se que a criança reproduza a mesma sequência imediatamente após a demonstração. Na ordem inversa a criança tem que memorizar a sequência vista, porém reproduzi-la na ordem inversa. Duas sequências de cada extensão serão executadas, a pontuação é dada pela extensão máxima desempenhada na ordem direta e inversa. Os pontos das ordens direta e inversa são gerados, cada repetição correspondendo à maior unidade de sequências reproduzidas precisamente em uma de duas tentativas. Este teste avalia tanto esboço visuoespacial na ordem direta como o executivo central na ordem inversa, ambos componentes de memória operacional, contudo, para informações visuais. Dados normativos para crianças brasileiras foram obtidos por Santos, Mello, Bueno e Dellatolas (2005).

Span de Dígitos. Este teste neuropsicológico que avalia tanto alça fonológica na ordem direta como o executivo central na ordem inversa, ambos componentes de memória operacional relacionados a informações verbais, foi retirado do subteste Memória de Dígitos da ZAREKI-R (von Aster \& Dellatolas, 2006). São apresentadas sequências crescentes de números que variam entre três e seis algarismos, na ordem direta e inversa, sendo quatro grupos de três sequências. $\mathrm{O}$ escore deste teste corresponde à maior quantidade de números que a criança conseguisse lembrar em uma sequência na ordem correta (span). Dados normativos Santos e Bueno (2003).

ZAREKI-R (von Aster \& Dellatolas, 2006) adaptada no Laboratório de Neuropsicologia da UNESP/Campus de Assis; dados normativos preliminares apresentados por Santos, Paschoalini e Molina (2006) e Santos e Silva (2008). A bateria tem duração aproximada de 30 minutos e é composta de 12 subtestes, descritos a seguir: 1) Enumeração de pontos: é composto de duas partes, cada uma com 3 itens. A primeira parte, sem pedido explícito de apontar com o dedo nem de contar em voz alta, permite ver se a criança utiliza espontaneamente estes processos. A segunda parte, com o pedido de contagem em voz alta e de marcação, permite examinar a sequência verbal, a sincronização entre esta sequência e a marcação, como os esquecimentos e as duplas contagens. Um só item tem uma disposição linear de pontos; as outras disposições mobilizam a atenção visuoespacial; 2) Contagem oral em ordem inversa: a criança deve contar inversamente a partir de um número apresentado pelo avaliador; 3) Ditado de números: a criança deve escrever, em numeral arábico, oito números apresentados oralmente; 4) Cálculo mental: pede-se para a criança resolver oito adições, oito subtrações e seis multiplicações que lhe são apresentadas oralmente; 5) Leitura de números: a criança deve ler oito números escritos em numeral arábico; 6) Posicionamento de números em escala vertical: É composto de duas partes. As escalas possuem um '0’ na base e um ' 100 ' no topo. Na primeira parte, as escalas possuem linhas horizontais em vários níveis e a criança deve apontar para a pequena linha horizontal que corresponde a 
três algarismos apresentado pelo examinador oralmente, e mais três formas escritas. Na segunda parte, as escalas verticais não apresentam pistas horizontais de localização dos algarismos, e a criança deve marcar com o lápis a posição correspondente a três algarismos apresentado oralmente, e outros três em forma escrita; 7) Memorização de dígitos: pede-se para a criança repetir, tanto na ordem direta quanto na ordem inversa, sequências crescentes de números que variam entre três e seis algarismos, sendo quatro grupos de três sequências, na ordem direta e inversa; sendo quatro grupos de três seqüências, na ordem direta e inversa. Comparação de números apresentados oralmente: oito pares de números são apresentados oralmente e a criança deve dizer qual dos dois números é maior; 9) Estimativa visual de quantidades Parte perceptiva: a criança deve dar uma estimativa, a partir da breve apresentação visual do número de itens presentes em quatro figuras; 10) Estimativa qualitativa de quantidades no contexto - Parte cognitiva contextual: a criança deve julgar proposições em termos quantitativos; 11) Problemas aritméticos apresentados oralmente: a criança deve resolver seis problemas aritméticos apresentados oralmente que aumentam gradativamente em dificuldade; e 12) Comparação de números escritos: dez pares de números são apresentados como numerais arábicos e a criança deve circular qual dos dois números é maior.

O estudo da validação de constructo da ZAREKI-R foi apresentado em Santos e Silva (2008) indicando correlações moderadas e altas entre 6 dos subtestes da ZAREKI-R e o Teste de Aritmética do TDE [enumeração de pontos $(\mathrm{r}=0,43)$; contagem oral em ordem inversa $(\mathrm{r}=0,44)$; ditado de números $(\mathrm{r}=0,69)$; cálculo mental $(\mathrm{r}=0,76)$; leitura de números $(\mathrm{r}=0,64)$; problemas aritméticos $(\mathrm{r}=0,55)]$ e ainda entre o escore total da ZAREKI-R e o Teste de Aritmética do TDE $(r=0,73)$.

\section{Análise Estatística}

Os dados obtidos foram analisados segundo o software do STATISTICA - versão 7. Statsoft Incorporation 1984-2004 (StatSoft, 2004). Para a análise com propostas inferenciais utilizou-se o teste-t de Student, tendo como variáveis dependentes os escores obtidos nos diversos testes neuropsicológicos e como variáveis independentes os grupos (CDA x SDA). Realizou-se também análise de variância (ANOVA) para medidas repetidas dos testes Span de Dígitos e Blocos de Corsi, tendo as mesmas variáveis dependentes e independentes, bem como a correlação de Pearson destas variáveis com os escores dos subtestes Cálculo Mental e Problemas Aritméticos. Os contrastes foram obtidos pelo teste post-hoc de Tukey e o nível de significância adotado foi o de 5\%. Os resultados não significantes não serão apresentados.

\section{Resultados}

A análise realizada com teste $t$ não evidenciou diferenças entre os grupos CDA e SDA para o Teste de Leitura do TDE e BCPR. No entanto, houve diferenças significativas nas Matrizes Coloridas de Raven, no Teste de Aritmética, no Teste de Escrita e no Escore Total do TDE, nos quais as crianças do grupo SDA apresentaram maiores escores que as crianças do grupo CDA (Ver Tabela 1). Para os testes Blocos de Corsi e Span de Dígitos (ordem direta x ordem inversa) foi utilizada ANOVA para medidas repetidas, para verificar efeito de grupo e ordem. A Tabela 1 apresenta os resultados obtidos pelos grupos.

Para o teste Blocos de Corsi, foi encontrado efeito de grupo $[F(1,28)=6,11 ; p=0,02]$, no qual, crianças SDA obtiveram maiores escores que o grupo CDA. Houve efeito de ordem dos blocos $[\mathrm{F}(1,28)=5,73 ; \mathrm{p}=0,02]$, no qual as crianças obtiveram um escore maior na ordem direta do que na ordem inversa. Não se constatou efeito de interação entre grupo e ordem $[F(1,28)=0,25 ; p=0,61]$.

Não foi observado efeito de grupo para a execução do teste Span de Dígitos, contudo, verificou-se que houve efeito de ordem dos números $[F(1,28)=45,68 ; p<0,0001]$, em que houve melhor desempenho na ordem direta do que na ordem inversa $(\mathrm{p}<0,0001)$. Foi constatado efeito de interação entre grupo e ordem $[\mathrm{F}(1,28)=5,29 ; \mathrm{p}=0,03]$, pois o grupo SDA apresentou maior escore do que o grupo CDA na ordem direta e menor escore que este grupo na ordem inversa.

No teste ZAREKI-R a análise dos resultados através do teste $t$ revelou efeito de grupo apenas para os subtestes Ditado de Números, Cálculo Mental, Problemas Aritméticos, nos quais os escores médios apresentados pelas crianças CDA foram significativamente menores que aqueles apresentados pelas crianças SDA. O mesmo foi observado para o Total da ZAREKI-R, conforme indicado na Tabela 2.

Uma análise utilizando o teste não-paramétrico de Kruskal-Wallis foi conduzida para controlar o efeito das múltiplas comparações. Os resultados confirmaram as análises realizadas: Ditado de Números $\left[\chi^{2}(1,30)=6,41 ; p=\right.$ $0,01]$, Cálculo Mental $\left[\chi^{2}(1,30)=11,30 ; p<0,01\right]$, Problemas Aritméticos $\left[\chi^{2}(1,30)=5,56 ; p=0,02\right]$ e Total $\left[\chi^{2}(1,30)=\right.$ $4,82 ; \mathrm{p}=0,03]$.

As análises de correlação pelo coeficiente de Pearson para ambos os grupos revelaram associações positivas baixas e moderadas entre as variáveis de memória e cálculo $(\mathrm{p}<0,05$, em todos os casos). As análises de correlação para as crianças CDA isoladamente exibiram o mesmo padrão, contudo, foram mais fortes, conforme especificado a seguir: 1) entre o subteste Cálculo Mental e Blocos de Corsi Ordem Direta, $\mathrm{r}=0,54$; e Ordem Inversa, $\mathrm{r}=0,52$; entre o mesmo subteste e Span de Dígitos Ordem Inversa, $\mathrm{r}=0,50$; entre o subteste Problemas Aritméticos e Blocos de Corsi Ordem Direta, $\mathrm{r}=0,47$; e Ordem Inversa, $\mathrm{r}=0,56$; entre o mesmo subteste e Span de Dígitos Ordem Inversa, $\mathrm{r}=0,75$.

Em análise qualitativa dos resultados obtidos no subteste Problemas Aritméticos para crianças CDA verificou-se que apenas quatro crianças realizaram a contagem como estratégia de resolução dos problemas, cinco relataram adotar a contagem mental e nove crianças solicitaram a repetição do enunciado de algum dos seis itens. Verificou-se, também, que as crianças exibiram três tipos distintos de respostas: $89,5 \%$ (17 crianças) acertaram pelo menos um item - o que denota tanto a compreensão do enunciado quanto a eficiência na realização dos procedimentos de cálculo; $21,1 \%$ (4 crianças) apresentaram erros na seleção da operação matemática apropriada em pelo menos um item - confundindo somas com 
Tabela 2. Desempenho [Média (DP)] nos subtestes e total da ZAREKI-R pelos grupos.

\begin{tabular}{|c|c|c|c|c|}
\hline & $\operatorname{SDA}(\mathrm{N}=11)$ & $\operatorname{CDA}(\mathrm{N}=19)$ & Teste $\mathrm{t}$ & $\mathrm{p}$ \\
\hline 1. Enumeração de Pontos & $3,63(0,67)$ & $3,31(0,82)$ & 1,09 & 0,28 \\
\hline 2. Contagem Oral em Ordem Inversa & $2,09(1,51)$ & $1,94(1,39)$ & 0,79 & 0,79 \\
\hline 3. Ditado de Números & $14,72(1,84)$ & $11,94(3,35)$ & 2,52 & $0,02 *$ \\
\hline 4. Cálculo Mental & $32,09(4,84)$ & $18,68(10,55)$ & 3,95 & $0,01 *$ \\
\hline Adição & $12,72(2,28)$ & $9,84(5,09)$ & 1,76 & 0,08 \\
\hline Subtração & $10,00(3,43)$ & $3,52(3,67)$ & 4,76 & $<0,0001^{*}$ \\
\hline Multiplicação & $9,36(2,76)$ & $5,63(4,25)$ & 2,59 & $0,01 *$ \\
\hline 5. Leitura de Números & $14,63(1,56)$ & $12,84(3,73)$ & 1,51 & 0,14 \\
\hline 6. Ordenação em escalas & $13,86(4,50)$ & $14,65(5,43)$ & $-0,40$ & 0,68 \\
\hline 7. Memória de dígitos & $18,90(2,07)$ & $17,26(5,58)$ & 0,93 & 0,35 \\
\hline 8. Comparação oral & $13,27(1,84)$ & $13,10(1,96)$ & 0,22 & 0,82 \\
\hline 9. Estimativa visual & $6,36(2,50)$ & $6,10(2,05)$ & 0,30 & 0,76 \\
\hline 10. Estimativa contextual & $10,18(4,04)$ & $9,15(4,86)$ & 0,58 & 0,56 \\
\hline 11. Problemas Aritméticos & $7,72(2,96)$ & $4,31(3,55)$ & 2,67 & $0,01 *$ \\
\hline 12. Comparação escrita & $18,18(5,40)$ & $17,89(2,78)$ & 0,19 & 0,84 \\
\hline Total** & $136,77(16,87)$ & $113,97(27,94)$ & 2,44 & $0,02 *$ \\
\hline
\end{tabular}

SDA - sem dificuldade em aritmética; CDA - com dificuldade em aritmética;

(*) $\mathrm{SDA}>\mathrm{CDA}(\mathrm{p}<0,05)$

(**) O Total foi computado somando-se os escores de todos os subtestes menos Memória de Dígitos.

subtração ou vice-versa; 73,7 \% (14 crianças) não emitiram respostas ou apresentaram respostas aleatórias (chutes) em algum dos itens. É importante ressaltar que das 19 crianças CDA apenas duas erraram todos os seis problemas do subteste, com respostas do tipo "não sei, não consigo"; enquanto 3 foram capazes de acertar os seis problemas aritméticos.

\section{Discussão}

O presente estudo teve como objetivo investigar quais aspectos da representação numérica e da memória operacional explicariam prejuízos em aritmética de crianças com Transtornos de Aprendizagem (TA) por meio da ZAREKI-R (von Aster \& Dellatolas, 2006), uma bateria específica para mensuração das habilidades matemáticas e especializada para o diagnóstico de Discalculia do Desenvolvimento (Shalev \& von Aster, 2008), e por tarefas de memória operacional (Blocos de Corsi, Span de Dígitos e BCPR).

Foram observadas diferenças significativas entre os grupos nas Matrizes Progressivas Coloridas de Raven, o SDA $(\mathrm{M}=61,7 ; \mathrm{DP}=19,2)$ e $\mathrm{CDA}(\mathrm{M}=45,3 ; \mathrm{DP}=11,8)$, embora ambos os valores fossem classificados como Grau III - Intelectualmente médio. $\mathrm{O}$ grupo SDA pode ser categorizado com médio superior (faixa de percentis entre 50-74), enquanto o grupo CDA no médio inferior (26-49). Portanto, essa diferença poderia em parte justificar algumas das diferenças encontradas no protocolo, pois, dificuldades em aritmética são associadas a um coeficiente de inteligência levemente mais baixo e este fator influencia na persistência dessas dificuldades (Shalev \& cols., 1998; Shalev \& cols., 2005).

No Teste de Desempenho Escolar - TDE, o grupo CDA foi significativamente inferior em Teste de Aritmética, Teste de Escrita e escore total, comprovando de forma objetiva que estas crianças apresentam dificuldades globais de aprendizagem. De fato, comorbidades entre o transtorno específico da habilidade em aritmética e outros transtornos de aprendizagem são mais comumente observadas do que a manifestação pura de discalculia (von Aster \& cols., 2007), sendo a dislexia a comorbidade mais associada à DD (Shalev \& cols., 2005).

Com relação ao estudo da memória operacional, no teste Blocos de Corsi as crianças do grupo SDA exibiram melhor desempenho que crianças CDA, corroborando os estudos de McLean e Hitch (1999) e Schuchardt e cols. (2008). Por outro lado, nas tarefas que avaliam alça fonológica - teste BCPR e Span de Dígitos - os grupos foram similares. Contudo, entre as crianças CDA as análises de correlações demonstraram associações entre memória operacional tanto visuoespacial quanto fonológica e as tarefas cálculo mental e problemas aritméticos da ZAREKI-R, possivelmente porque ambas as tarefas são apresentadas oralmente, o que aumentaria as 
demandas da alça fonológica (Swanson, 1993). Tanto no teste Blocos de Corsi, quanto no Span de Dígitos, as crianças foram melhores na ordem direta do que na ordem inversa, assim como em estudos prévios (Santos \& Bueno, 2003; Santos \& cols., 2005), tendo em vista que a capacidade do componente executivo central, avaliado na ordem inversa, é menor do que dos armazenadores.

Em termos de representação numérica (McCloskey \& cols., 1985), as crianças CDA, de acordo com as hipóteses do presente estudo, apresentaram déficits na ZAREKI-R, tanto em processamento numérico como em cálculo. No teste Ditado de Números as crianças CDA apresentaram prejuízos leves em relação às crianças SDA, revelando dificuldades na transcodificação entre os sistemas verbal e arábico de números, isto é, da forma falada para a forma escrita (von Aster \& Shalev, 2007; Dellatolas \& cols., 2000). Em ambos os subtestes, as crianças CDA apresentaram escores inferiores às crianças SDA, com prejuízo moderado no subteste Cálculo Mental (menos dois desvios-padrão).

$\mathrm{Na}$ análise qualitativa do desempenho do grupo CDA no subteste Problemas Aritméticos, o grupo CDA não apóia a hipótese de falha na produção numérica, pois erros desta natureza foram raros $(<2 \%)$, no entanto, $21,1 \%$ das crianças apresentaram erros na escolha do procedimento, o que corrobora falha no processamento dos símbolos ou palavras operacionais, e na execução de procedimentos de cálculos aritméticos (McCloskey \& cols., 1985). Por outro lado, 73,7\% das crianças não foram capazes de responder a pelo menos um dos seis itens do subteste, confirmando um atraso significativo na consolidação dos sistemas numéricos simbólicos e na aquisição do pensamento aritmético em crianças CDA, habilidades estas esperadas na transição entre pré-escola e início do Ensino Fundamental (Mundy \& Gilmore, 2009; von Aster \& Shalev, 2007). Além disso, os erros apresentados pelas crianças CDA foram mais significativos quando a incógnita estava nas duas posições iniciais e não no resultado, o que corrobora com os estudos de Haydu e cols. (2006) e Fayol (1996). É importante destacar que o grupo CDA obteve uma correlação alta $(\mathrm{r}=0,75)$ entre problemas aritméticos e o span de dígitos em ordem inversa sugerindo que a resolução de tarefas desta natureza depende substancialmente da capacidade para memorizar as informações contidas no enunciado (Baddeley, 1986; Schuchardt \& cols. 2008).

Em contraste com os dados normativos preliminares coletados no Brasil (Santos \& cols., 2006; Santos \& Silva, 2008), a média do escore total da ZAREKI-R de crianças CDA foi um desvio-padrão e meio abaixo do esperado para crianças com desenvolvimento típico de mesma idade e também em relação às crianças SDA do presente estudo. Koumoula e cols. (2004) adotaram este parâmetro para diagnóstico de DD em seu estudo; mais recentemente Rotzer e cols. (2009) acrescentaram que o diagnóstico de DD poderia ser estabelecido tanto pelo prejuízo em três subtestes como no total ZAREKI-R. Portanto, pode-se sugerir que no presente estudo o grupo CDA, apresentou um perfil compatível com DD, caracterizado por prejuízos na representação numérica tanto no que concerne ao cálculo quanto ao processamento numérico simbólico (Rotzer \& cols., 2009).
Do ponto de vista clínico também é importante destacar que crianças com prejuízos específicos em matemática (grupo CDA) exibiram um perfil de dificuldades na representação numérica diferente das crianças com transtornos em leitura e escrita (grupo SDA). Portanto crianças de salas de reforço, muitas vezes assistidas por um mesmo programa de intervenção ou estimulação por serem consideradas um grupo homogêneo podem apresentar dificuldades ou transtornos de aprendizagem com perfis muito distintos ou em comorbidade, necessitando, consequentemente de intervenções distintas. No presente estudos tarefas especificas (ditado, cálculo e problemas aritméticos) foram particularmente sensíveis para distinguir os grupos CDA e SDA. Estes resultados ressaltam a importância de que crianças com dificuldade de aprendizagem sejam encaminhadas para avaliação neuropsicológica que tem se mostrado um procedimento diagnóstico confiável para a identificação das crianças com DD e de potenciais comorbidades (Santos, Silva, Ribeiro, \& Kikuchi, 2010).

O estudo apresenta como limitação o tamanho reduzido da amostra. Contudo, a caracterização e seleção criteriosa da amostra pode ser vista como uma vantagem do estudo, pois garantiram a priori a presença de TA avaliada pela escola e a presença de dificuldade em aritmética, mensurada de forma objetiva por meio do TDE. Enquanto, informações aferidas pela anamnese permitiram controlar variáveis que poderiam confundir a interpretação das análises. Além disso, tratamentos estatísticos não-paramétricos foram utilizados para análises confirmatórias. Portanto, os resultados obtidos no estudo se mostram confiáveis.

Neste estudo, as crianças CDA exibiram um nível intelectual médio inferior, enquanto as crianças SDA possuíam um nível intelectual médio superior. Apresentaram também déficits em memória operacional para informações visuoespaciais. Com relação à representação numérica, exibiram déficits tanto em processamento numérico quanto em cálculo, compatíveis com DD. A ZAREKI-R foi eficaz na detecção de pontos fracos relacionados às habilidades matemáticas $\mathrm{e}$ deve integrar a avaliação da DD.

\section{Referências}

Adams, J. W., \& Hitch, G. J. (1997). Working memory and children's mental addition. Journal of Experimental Child Psychology, 67 (1), 21-38.

Almeida, P. M., \& Wickerhauser, H. (1991). O Critério ABA/ ABIPEME - em Busca de Uma Atualização. Um estudo e uma proposta submetidos à $\mathrm{ABA}$ e à $\mathrm{ABIPEME}$. Documento de circulação restrita da ABA e da ABIPEME, São Paulo.

American Psychiatric Association. (2002). DSM-IV-TR: Manual diagnóstico e estatístico de transtornos mentais (4a ed. texto revisado). Porto Alegre: Artmed.

Baddeley, A. D. (1986). Working memory. Oxford: Oxford University Press.

Baddeley, A. D. (2000). The episodic buffer: a new component of working memory? Trends in Cognitive Sciences, 4 (11), 417-23.

Baddeley, A. D., \& Hitch, G. J. (1974). Working Memory. In: Bower, G (Ed.). The Psychology of Learning and Motivation (pp. 44-90). London: Academic Press. 
Chahon, M. (2006). Metacognição e resolução de problemas aritméticos verbais: teoria e implicações pedagógicas. Revista do Departamento de Psicologia UFF, 18(2), 163-176.

Ciasca, S. M. (1994). Distúrbios e Dificuldades de Aprendizagem em crianças: análise do diagnóstico interdisciplinar (Tese de Doutorado não publicada). Universidade Estadual de Campinas, Campinas.

Dehaene, S. (2001). Précis of the number sense. Mind \& Language, 16, 16-36.

Dellatolas, G., von Aster, M., Braga, L.W., Meier, M., \& Deloche, G. (2000). Number processing and mental calculation in school children aged 7 to 10 years: A transcultural comparison. European Child and Adolescent Psychiatry, 9(2), 102-110.

Dias, T. L., Enumo, S. R. F., Turini, F. A., \& Ferrão, E. S. (2003). Dificuldade de Aprendizagem: avaliação do desempenho em provas acadêmicas e cognitivas. In S. R. F. Enumo, S. S. Queiroz, \& A. Garcia (Eds.), Desenvolvimento humano e aprendizagem: algumas análises e pesquisas (pp. 59-78). São Paulo: Casa do Psicólogo.

Fayol, M. (1996). A criança e o número: da contagem à resolução de problemas. Porto Alegre: Artes Médicas.

Feigenson, L., Dehaene, S., \& Spelke, E. (2004). Core systems of number. Trends in Cognitive Sciences, 8, 307-314.

Geary, D. C. (1995). Reflections of evolution and culture in children's cognition: implications for mathematical development and instruction. American Psychologist, 50 (1), 24-37.

Geary, D. C. (2000). From infancy to adulthood: The development of numerical abilities. European Child and Adolescent Psychiatry, 9(2), 11-16.

Gross-Tsur, V., Manor, O., \& Shalev, R. S. (1996). Developmental dyscalculia: prevalence and demographic features. Developmental Medicine and Child Neurology, 38, 25-33.

Haskell, S. H. (2000). The determinants of arithmetic skills in young children: some observations. European Child \& Adolescent Psychiatry, 9(2), 77-86.

Haydu, V. B., Costa, L. P., \& Pullin, E. M. M. P. (2006). Resolução de problemas aritméticos: efeitos de relações de equivalência entre três diferentes formas de apresentação dos problemas. Psicologia: Reflexão e Crítica, 19(1), 44-52.

Koumoula, A., Tsironi, V., Stamouli, V. Bardani, I., Siapati, S., Graham, A., \& cols. (2004). An epidemiological study of number processing and mental calculation in Greek school children. Journal of Learning Disabilities, 37(5), 377- 388.

Lewis, C., Hitch, G. J., \& Walker, P. (1994). The prevalence of specific arithmetic difficulties and specific reading difficulties in 9- to 10-year-old boys and girls. Journal of Child Psychology and Psychiatry, 35, 283-292.

McCloskey, M., Caramazza, A., \& Basili, A. (1985). Cognitive mechanisms in number processing and calculation: Evidence from dyscalculia. Brain and Cognitive, 4, 171-196.

Mclean, J. F., \& Hitch, G. J. (1999). Working memory impairments in children with specific arithmetic learning difficulties. Journal of Experimental Child Psychology, 74(3), 240-260.

Mundy, E.; Gilmore, C. K. (2009). Children's mapping between symbolic and nonsymbolic representations of number. Journal of Experimental Child Psychology, 103, 490-502.

Noël, M.-P. (2005). Rôle de la mémoire de travail danos l'apprentissage du cálculo. In A. Van Hout, C. Meljac \& J.P. Fischer (Eds.), Troubles du cálculo e dyscálculoies chez l'criança (pp. 186-194). Paris: Masson.
Organização Mundial da Saúde (1993). Classificação de transtornos mentais e de comportamento da CID-10: descrições clínicas e diretrizes diagnósticas. Porto Alegre: Artes Médicas.

Raven, J. C. (1992). Teste de Matrices Progresivas: Escala especial. Buenos Aires: Editorial Paidos.

Rotzer, S., Loenneker, T., Kucian, K., Martin, E., Klaver, P., \& von Aster, M. (2009). Dysfunctional neural network of spatial working memory contributes to developmental dyscalculia. Neuropsychologia, 47(13), 2859-2865.

Rubinsten, O., \& Henik, A. (2009). Developmental Dyscalculia: heterogeneity might not mean different mechanisms. Trends in Cognitive Sciences, 13(2), 92-99.

Santos, F. H., \& Bueno, O. F. A. (2003). Validation of the Brazilian children's test of pseudoword repetition in portuguese speakers aged 4-10 years. Brazilian Journal Medical Biology Research, 36, 1533-1547.

Santos, F. H., Mello, C. B., Bueno, O. F. A., \& Dellatolas, G. (2005). Cross-cultural differences for three visual memory tasks in Brazilian children. Perceptual and Motor Skills, 101, 421- 433.

Santos, F. H., Paschoalini, B., \& Molina, J. (2006). Novos instrumentos para avaliação de habilidades matemáticas em crianças. In: A. L. Sennyey; L. I .Z. Mendonça; B. B. G. Schlecht; E. F. Santos, \& E. C. Macedo. (Eds.), Neuropsicologia e Inclusão (1ed) (pp. 69-80). São Paulo: Artes Médicas, v.1.

Santos, F. H., \& Silva, P. A. (2008). Avaliação da discalculia do desenvolvimento: uma questão sobre o processamento numérico e o cálculo. In: A. L. Sennyey, F. C. Capovilla, \& J. M. Montiel (Eds.), Transtornos de Aprendizagem: da Avaliação à reabilitação (1ed) (pp. 125-137). São Paulo: Artes Médicas.

Santos, F. H., Silva, P. A., Ribeiro, F. S., \& Kikuchi, R. S. (2010). Recomendações para professores sobre o "Transtorno da Matemática". Revista Sinpro-Rio, nº 05, 19-33.

Schuchardt, K., Maehler, C., \& Hasselhorn, M. (2008). Working memory deficits in children with specific learning disorders. Journal of Learning Disabilities, 41 (6), 514-523.

Shalev, R. S. (2004). Developmental dyscalculia: Review. Journal of Child Neurology, 19, 765-771.

Shalev, R. S., Auerbach, J., Manor, O., \& Gross-Tsur, V. (2000). Developmental dyscalculia: prevalence and prognosis. European Child \& Adolescent Psychiatry, 9(2), 58- 64.

Shalev, R. S., Manor, O., Auerbach, J., \& Gross-Tsur, V. (1998). Persistence of developmental dyscalculia: what counts? Results from a three year prospective follow-up study. Journal of Pediatrics, 133, 358-362.

Shalev, R. S., Manor, O., \& Gross-Tsur, V. (2005). Developmental dyscalculia: a prospective six-year follow-up. Developmental Medicine and Child Neurology, 47, 121-125.

Shalev, R. S., \& von Aster, M. G. (2008). Identification, classification, and prevalence of developmental dyscalculia. Encyclopedia of Language and Literacy Development (pp. 1-9). London, ON: Canadian Language and Literacy Research Network. Retirado em 05/12/2008, de http://www.literacyencyclopedia.ca/pdfs/ topic.php?topId $=253$.

StatSoft, Inc. (2004). STATISTICA (data analysis software system), version 7.

Stein, L. M. (1994). TDE: Teste de Desempenho Escolar: manual para aplicação e interpretação. São Paulo: Casa do Psicólogo.

Swanson, H. L. (1993). Working Memory in Learning Disability Subgroups. Journal of Experimental Child Psychology, 56(1), 87-114. 
von Aster, M., \& Dellatolas, G. (2006). ZAREKI-R - Batterie pour l'évaluation du traitement des nombres et $d u$ calcul chez l'enfant. Paris: ECPA.

von Aster, M. G., \& Shalev, R. S. (2007). Number development and developmental dyscalculia. Developmental Medicine \& Child Neurology, 49, 868-873.

von Aster, M. G., Schweiter, M., \& Weinhold Zulauf, M. (2007). Rechenstörungen bei Kindern. Vorläufer, Prävalenz und psychische Symptome. [Developmental dyscalculia: Precursors, prevalence and co morbidity.] Zeitschrift fürEntwicklungs Pädagogik, 39, 85-96.

Recebido em 20.03.2009 Primeira decisão editorial em 06.05.2010 Versão final em 07.06.2010 Aceito em 11.06.2010

\section{CONGRESSO DE PSICOLOGIA SOCIAL E DO TRABALHO}

Período: 13 de Maio - 13 Maio 2011

Local: Poços de Caldas, MG, BR) 